\title{
Pulmonary hypertension due to left heart disease: diagnostic value of pulmonary artery distensibility
}

\author{
Geoffrey C. Colin ${ }^{1} \cdot$ Guillaume Verlynde ${ }^{1} \cdot$ Anne-Catherine Pouleur ${ }^{2} \cdot$ Bernhard L. Gerber $^{2} \cdot$ Christophe Beauloye $^{2} \cdot$ \\ Joelle Kefer ${ }^{2} \cdot$ Emmanuel Coche $^{1} \cdot$ Jean-Luc Vachiéry ${ }^{3} \cdot$ Pierre Alain Gevenois ${ }^{4} \cdot$ Benoit Ghaye $^{1}$
}

Received: 30 December 2019 / Revised: 28 March 2020 / Accepted: 14 May 2020

(C) European Society of Radiology 2020

\begin{abstract}
Objectives To evaluate how pulmonary artery (PA) distensibility performs in detecting pulmonary hypertension due to left heart disease (PH-LHD) in comparison with parameters from ungated computed tomography (CT) and echocardiography.

Methods One hundred patients ( 79 men, mean age $=63 \pm 17$ years) with either severe heart failure with reduced ejection fraction (HFrEF), aortic stenosis, or primary mitral regurgitation prospectively underwent right heart catheterization, ungated CT, ECG-gated CT, and echocardiography. During the ECG-gated CT, the right PA distensibility was calculated. In ungated $\mathrm{CT}, \mathrm{dPA}, \mathrm{dPA} / \mathrm{AA}$, the ratio of $\mathrm{dPA}$ to the diameter of the vertebra, segmental PA diameter, segmental PA-to-bronchus ratio, and the main PA volume were measured; the egg-and-banana sign was recorded. During echocardiography, the tricuspid regurgitation (TR) gradient was measured. The areas under the ROC curves (AUC) of these signs were computed and compared with DeLong test. Correlation between PA distensibility and PA pressure (PAP) was investigated through Pearson's coefficient.

Results PA distensibility was lower in patients with PH than in those without PH $(11.4$ vs. $21.2 \%, p<0.001)$ and correlated negatively with mean PAP $(r=-0.72, p<0.001)$. Age, PA size, and mean PAP were independent predictors of PA distensibility. PA distensibility $<18 \%$ detected PH-LHD with $96 \%$ sensitivity and $73 \%$ specificity; its AUC was 0.92, larger than that of any other sign at ungated CT and TR gradient (AUC ranging from 0.54 to 0.83, DeLong: $p$ ranging from 0.020 to $<0.001)$.

Conclusion PA distensibility on an ECG-gated CT can detect PH-LHD better than the parameters reflecting PA dilatation in ungated CT or TR gradient in the echocardiography of patients with severe HFrEF, aortic stenosis, or mitral regurgitation.

Key Points

- In left heart disease, pulmonary artery distensibility is lower in patients with PH than in those without pulmonary hypertension (11.4 vs. $21.2 \%, p<0.001)$.

- In left heart disease, pulmonary artery distensibility detects pulmonary hypertension with an area under the receiver operating curve of 0.92 .

- In left heart disease, the area under the receiver operating curve of pulmonary artery distensibility for detecting pulmonary hypertension is larger than that of all other signs at ungated CT (p from 0.019 to $<0.001$ ) and tricuspid regurgitation gradient at echocardiography $(p=0.020)$.
\end{abstract}

Keywords Pulmonary hypertension $\cdot$ Computed tomography $\cdot$ Echocardiography $\cdot$ Heart failure

Geoffrey C. Colin

gc.colin.md@gmail.com; geoffrey.colin@uclouvain.be

1 Division of Radiology, Cliniques Universitaires Saint-Luc,

Université catholique de Louvain (UCL), Avenue Hippocrate 10, 1200 Brussels, Belgium
2 Division of Cardiology, Cliniques Universitaires Saint-Luc, Université catholique de Louvain (UCL), Brussels, Belgium

3 Department of Cardiology, Hôpital Erasme, Université libre de Bruxelles (ULB), Brussels, Belgium

4 Department of Radiology, Hôpital Erasme, Université libre de Bruxelles (ULB), Brussels, Belgium 


\begin{tabular}{|c|c|}
\hline \multicolumn{2}{|c|}{ Abbreviations } \\
\hline ABR & Pulmonary artery to bronchus ratio \\
\hline AUC & $\begin{array}{l}\text { Area under the receiver operating } \\
\text { characteristic curve }\end{array}$ \\
\hline dPA & Diameter of the main pulmonary artery \\
\hline dPA/AA & $\begin{array}{l}\text { Diameter of the main pulmonary artery } \\
\text { to the diameter of the ascending aorta ratio }\end{array}$ \\
\hline $\mathrm{dPA} / \mathrm{V}$ & $\begin{array}{l}\text { Diameter of the main pulmonary artery to } \\
\text { the diameter of the vertebral body ratio }\end{array}$ \\
\hline HFrEF & Heart failure with reduced injection fraction \\
\hline PA & Pulmonary artery \\
\hline PAP & Pulmonary artery pressure \\
\hline PAWP & Pulmonary artery wedge pressure \\
\hline PH & Pulmonary hypertension \\
\hline PH-LDH & Pulmonary hypertension due to left heart disease \\
\hline TR & Tricuspid regurgitation \\
\hline
\end{tabular}

\section{Introduction}

Pulmonary hypertension $(\mathrm{PH})$ due to left heart disease (PHLHD) is defined as a mean pulmonary artery (PA) pressure greater than or equal to $25 \mathrm{mmHg}$; it is associated with a PA wedge pressure greater than $15 \mathrm{mmHg}$ as measured by right heart catheterization [1]. PH-LHD carries a worse prognosis by increasing the right ventricular afterload [2-4]. Patients with left heart disease are routinely evaluated using echocardiography that provides a noninvasive estimation of pulmonary pressure. The tricuspid regurgitation (TR) gradient computed from the TR velocity by the continuous wave spectral Doppler is the more routinely used method for detecting PH at echocardiography [1]. However, possible diagnostic inaccuracies of such TR gradient have been widely reported, due to weak or absent TR jet at spectral Doppler, lack of parallel alignment of the ultrasound beam to the TR jet, and intraday variation due to patient-related factors including blood volume status [5]. Thus, despite its invasiveness, right heart catheterization remains the standard of reference in the diagnosis and severity assessment of $\mathrm{PH}[1]$.

Various parameters have been reported for noninvasive detection of $\mathrm{PH}$ in ungated chest computed tomography (CT), mainly based on PA measurements, including the diameter of the main PA (dPA) [6-16], the ratio between $\mathrm{PPA}$ and the diameter of the ascending aorta (dPA/AA) [8-18], the ratio between $\mathrm{PPA}$ and the diameter of the thoracic vertebra (dPA/ V) [17], the volume of the main PA [19,20], the segmental PA diameter [17], the segmental PA-to-bronchus ratio (ABR) [7, $12,17]$, and the so-called egg-and-banana sign $[12,15]$. These parameters have been separately assessed and are all of limited value unless combined with echocardiography [17].

In ECG-gated CT, the PA distensibility-defined as the relative difference between the systolic and diastolic PA cross-sectional areas-performs well in detecting PH [21].
Nevertheless, the diagnostic value of PA distensibility in PH-LHD, as well as its diagnostic value when compared with ungated CT and echocardiography, remains unknown. In addition, most of the CT vascular parameters have been evaluated in patients with precapillary or mixed types of $\mathrm{PH}$, although PH-LHD is the most common [2]. The purpose of this study was, therefore, to assess the respective values of CT vascular parameters in a group of patients with PH-LHD, considering right heart catheterization as the independent method of reference.

\section{Materials and methods}

\section{Study group}

Our local ethics committee approved the protocol of this prospective investigation. All patients gave written informed consent. Between October 2015 and April 2019, patients who were scheduled to undergo clinically indicated right heart catheterization for either heart failure with reduced ejection fraction (HFrEF) considered for heart transplantation, aortic stenosis considered for transcatheter aortic valve implantation, or primary mitral regurgitation considered for surgery were invited to participate to this investigation. Patients with contraindications to the injection of an iodinated contrast medium (i.e., intolerance to contrast medium or renal insufficiency with an estimated glomerular filtration rate of $<30 \mathrm{~mL} / \mathrm{min} /$ $1.73 \mathrm{~m}^{2}$ ), except those under dialysis, were not invited to participate. Patients younger than 30 year old, with congenital heart disease or acute heart failure defined as rapid onset, or with worsening of symptoms and/or signs of heart failure were also not invited to participate [22]. One hundred and three patients were invited and consented to participate.

Of the 103 enrolled patients, three patients were excluded because of major CT artifacts in their ECG-gated CT due to extrasystolic beats in two patients and severe arrhythmia during RHC in one patient, resulting in incomplete data. Thus, our final study group consisted of 100 patients: 79 men (79\%), mean age of $63 \pm 17$ years, with either HFrEF (54 patients), aortic stenosis (31 patients), or primary mitral regurgitation (15 patients). The median time intervals between $\mathrm{CT}$ and right heart catheterization and between echocardiography and right heart catheterization were 1 day (interquartile ranges, $0-1$ day and 1-2 days, respectively).

\section{Right heart catheterization and echocardiography}

Right heart catheterization was performed using the standard technique, with measurements of mean PA pressure (PAP), PA wedge pressure (PAWP), cardiac output, and computation of pulmonary vascular resistance [1]. PH-LHD was defined as mean PAP $\geq 25 \mathrm{mmHg}$ and PAWP $>15 \mathrm{mmHg}$. Combined 
pre- and post-capillary PH was defined as mean PAP $\geq 25 \mathrm{mmHg}$, PAWP $>15 \mathrm{mmHg}$, associated with pulmonary vascular resistance $>3$ Wood Units, and/or diastolic pulmonary gradient (diastolic PAP - mean PAWP) $\geq 7 \mathrm{mmHg}$ [1]. Echocardiography was performed on IE33 or Epic sonographs (Philips Medical Systems, Cleveland, $\mathrm{OH}$ ) by experienced cardiologists (all $>10$ years of experience). In accordance with the most recent guidelines, the TR gradient was used to detect $\mathrm{PH}[1]$.

\section{ECG-gated and ungated CT}

CT examinations were performed using a 256-detector row scanner (ICT, Philips Healthcare). First-pass perfusion images were acquired on a single transversal slice through both ventricles with the following parameters: slice thickness, $10 \mathrm{~mm}$; rotation time, $270 \mathrm{~ms}$; tube potential, $100 \mathrm{kV}$; and tube current, $20 \mathrm{~mA}$. The contrast injection protocol consisted of $40 \mathrm{~mL}$ of $400 \mathrm{mg}$ iodine $/ \mathrm{mL}$ iodinated contrast medium (Iomeron®, Iomeprol, Bracco Diagnostics) injected at a flow rate of $5 \mathrm{~mL} / \mathrm{s}$ through a right antecubital vein via an 18 -gauge catheter and followed by $20 \mathrm{~mL}$ of saline bolus chaser at $5 \mathrm{~mL} / \mathrm{s}$. The acquisition was discontinued as soon as the contrast density visually decreased in the descending aorta.

Immediately thereafter, a second acquisition consisted of a retrospective ECG-gated cardiac $\mathrm{CT}$ after an additional injection of $60 \mathrm{~mL}$ of contrast medium at $2 \mathrm{~mL} / \mathrm{s}$, with bolus tracking in the ascending aorta. The acquisition was debuted when the attenuation had increased to $180 \mathrm{HU}$. The parameters were collimation, $128 \times 0.625 \mathrm{~mm}$; rotation time, $270 \mathrm{~ms}$; tube potential from 100 to $120 \mathrm{kV}$, depending on the patient's weight; and tube current from 300 to $800 \mathrm{mAs}$, depending on the automatic tube current modulation. All contrast medium injections were performed with a power injector (Medrad Stellant, Bayer Healthcare), and CM was heated using syringe heating.

Finally, a third acquisition consisted of a thoracoabdominal scan, $60 \mathrm{~s}$ after the end of the second acquisition with the following parameters: tube potential, $120 \mathrm{kV}$; tube current from 40 to $150 \mathrm{mAs}$, depending on automatic tube current modulation. The effective delivered radiation dose was calculated as follows: dose-length product provided by the scanner unit multiplied by 0.017 [23]. The average radiation exposure delivered by ungated $\mathrm{CT}$ and ECG-gated $\mathrm{CT}$ acquisitions was $3.4 \pm 0.9 \mathrm{mSv}$ and $11.5 \pm 1.8 \mathrm{mSv}$, respectively.

The CT images were analyzed with commercially available software (CT viewer and CT cardiac viewer, Philips Healthcare) by observer $\mathrm{Nr} 1$ (G.V.), who had 3 years of experience in cardiac imaging, and blinded to right heart catheterization results. In ungated $\mathrm{CT}, \mathrm{dPA}, \mathrm{dPA} / \mathrm{AA}$, and $\mathrm{dPA} / \mathrm{V}$ were measured as previously reported [8-18]. The small segmental PA diameter was measured in four lobes on axial sections: the apical segment of the right upper lobe, the posterior segment of the left upper lobe, the posterior basal segment of the right lower lobe, and the posterior basal segment of the left lower lobe [17]. The average segmental PA diameter was calculated from these four measurements. The segmental ABR was calculated as the segmental PA diameter divided by the small outer diameter of the adjacent bronchus and was considered as enlarged if $>1.25$ [17]. Segmental ABR was scored from 0 to 4 , on the basis of the number of enlarged ABR in the four lobes. The main PA volume was calculated as follows: a center line was traced from the center of the pulmonary valve to the main PA bifurcation. The length of this center line was considered to be the main PA length. The PA cross-sectional area was measured at each 10-mm segment of the center line, and a mean area was computed. The PA volume was calculated as mean PA area multiplied by the PA length, expressed in milliliter. The egg-and-banana sign was present when the main PA was visible at the bottom level of the aortic arch [15].

For ECG-gated CT, the right PA distensibility was measured as proposed by Revel et al [21]. From a parasagittal view, the cross-sectional area of the right PA was obtained after a double oblique adjustment on axial and coronal views, $1 \mathrm{~cm}$ distal to the main PA division, and manually delineated every $10 \%$ of the cardiac cycle. PA distensibility, expressed as a percentage, was defined as the following ratio: (maximal cross-sectional area minus minimal cross-sectional area)/maximal cross-sectional area [21]. In addition, the dPA and dPA/ AA were measured on axial slices for every $10 \%$ of the R-R interval in the same way as described for ungated CT. The maximal and minimal values for $\mathrm{APA}$ and $\mathrm{DPA} / \mathrm{AA}$ were noted. Examples of CT measurements are illustrated in Fig. 1. In order to assess interobserver agreement, PA distensibility was independently calculated by observer $\mathrm{Nr} 2$ (G.C.), who had 8 years of experience in cardiac imaging. In order to assess intraobserver agreement, observer $\mathrm{Nr} 1$ (G.V.) redid the PA distensibility calculation more than 1 month later.

\section{Statistical analysis}

Continuous variables were expressed as means \pm standard deviations or as medians with interquartiles. Categorical variables were expressed as counts and proportions. Groups were compared using Student's unpaired $t$ test or $\chi^{2}$ test when appropriate. A non-parametric test (Kruskal-Wallis) was used to compare the median values of ABR. Interobserver agreement for PA distensibility was assessed by using Lin's concordance correlation coefficient. The relationships between mean PA pressure, CT parameters, and TR gradient were tested using linear regression analysis and Pearson's correlation coefficient. The Pearson correlation coefficient was used to assess the relationship of PA distensibility with patient characteristics, PA size, and hemodynamics. Multivariable analysis 

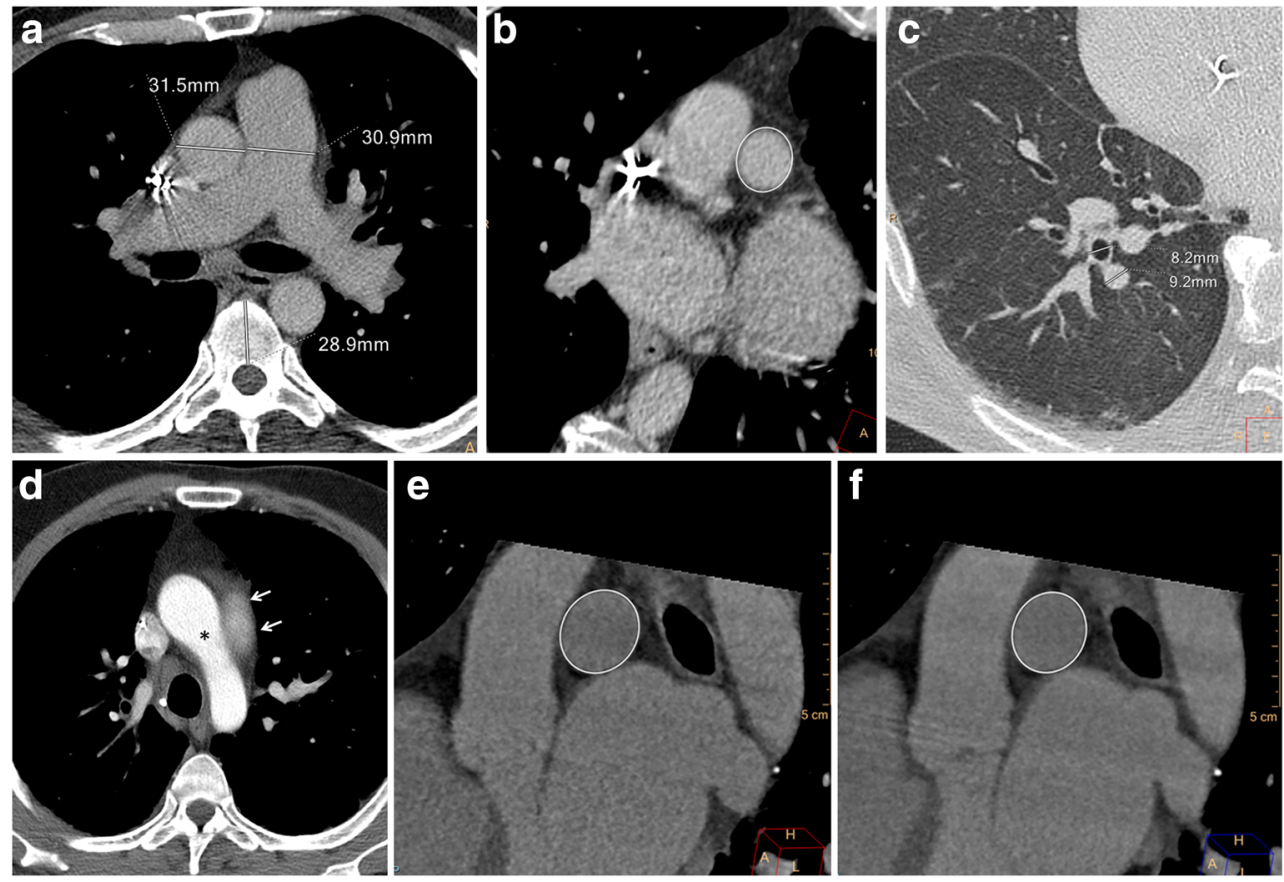

Fig. 1 Computed tomography measurements in a 57-year-old man with ischemic dilated cardiomyopathy and severe left ventricular systolic dysfunction. a At ungated CT, the pulmonary artery diameter was $30.9 \mathrm{~mm}$, the aortic diameter was $31.5 \mathrm{~mm}$, and the diameter of the vertebral body was $28.9 \mathrm{~mm}$. b Measurement of the pulmonary artery area after double oblique reformation. $\mathbf{c}$ Measurement of the right posterior basal segmental artery-to-bronchus ratio. The segmental artery diameter was $9.2 \mathrm{~mm}$

using backward elimination was performed with all variables associated in univariable analysis $(p<0.10)$.

The value of CT parameters and TR gradient for diagnosing PH-LHD was assessed through the area under the receiver operating characteristic curves (AUCs). The AUCs were compared against 0.5 and one another using the DeLong test. A $p$ value $<0.05$ was considered to be statistically significant. All statistical analyses were performed using NCSS 12 Statistical Software (LLC).

\section{Results}

At right heart catheterization, 56 patients had $\mathrm{PH}$, while 44 patients had no PH. All 56 patients with $\mathrm{PH}$ had PHLHD. Among them, 27 patients had associated combined pre- and postcapillary PH with pulmonary vascular resistance $>3$ Wood Units and among these, twelve patients had also elevated diastolic pulmonary gradient $\geq 7 \mathrm{mmHg}$. Eight patients $(8 \%)$ had chronic lung disease (five patients with chronic obstructive pulmonary disease, one patient with hypersensitivity pneumonitis, one patient with pulmonary fibrosis, and one patient with nonspecific interstitial pneumonia). and the adjacent bronchus diameter was $8.2 \mathrm{~mm}$, allowing an artery-tobronchus ratio of 1.12. d Egg-and-banana sign consisting in the visibility of the pulmonary artery (arrows) at the level of the aortic arch $(*)$. At gated CT, the right pulmonary artery distensibility was calculated at $11 \%$; the right pulmonary cross-sectional area was measured at $570 \mathrm{~mm}^{2}$ in systole (30\% of the RR interval, e) and $508 \mathrm{~mm}^{2}$ in diastole $(0 \%$ of the RR interval, f)

The patient characteristics and echocardiography, hemodynamics, ungated CT, and ECG-gated CT parameters of patients with and without $\mathrm{PH}$ are compared in Table 1.

There was no significant difference between patients with and those without $\mathrm{PH}$ regarding age, gender, body mass index, ischemic heart disease, and chronic lung disease. Patients with PH had higher heart rate $(p \leq 0.001)$ and had more frequently atrial fibrillation $(p=0.014)$ than those without $\mathrm{PH}$. Among CT parameters, dPA, dPA/AA ratio, and $\mathrm{dPA} / \mathrm{V}$ ratio, as well as main $\mathrm{PA}$ mean crosssectional area and volume, were significantly larger in patients with $\mathrm{PH}$ as compared with those in patients without PH (all $p<0.001$ ). By opposition, main PA length, segmental PA diameter, median segmental ABR score, and egg-and-banana sign were not significantly different between both groups (all $p>0.05$ ). The AUCs of each parameter assessed in ungated CT, ECG-gated CT, and echocardiography for detecting $\mathrm{PH}$ are listed and compared with 0.5 in Table 2 . The correlations between these parameters and mean PA pressure are also listed in Table 2.

PA distensibility $<18 \%$ detected PH-LHD with $96 \%$ sensitivity and $73 \%$ specificity. Its AUC (0.92) was significantly larger than that of the TR gradient, dPA/AA (Fig. 2), dPA, main PA cross-sectional area, and all other ungated CT 
Table 1 Patient characteristics, hemodynamics, echocardiography, and CT parameters

\begin{tabular}{|c|c|c|c|c|}
\hline & Whole study group $(n=100)$ & Patients without $\mathrm{PH}(n=44)$ & Patients with PH $(n=56)$ & $p$ value \\
\hline \multicolumn{5}{|l|}{ Patient characteristics } \\
\hline Age, years & $63 \pm 17$ & $61 \pm 15$ & $64 \pm 17$ & 0.337 \\
\hline Men & $79(79)$ & $34(77)$ & $45(80)$ & 0.706 \\
\hline BMI, $\mathrm{kg} / \mathrm{m}^{2}$ & $26 \pm 5$ & $26 \pm 5$ & $26 \pm 4$ & 0.653 \\
\hline $\mathrm{BSA}, \mathrm{m}^{2}$ & $1.89 \pm 0.22$ & $1.89 \pm 0.20$ & $1.89 \pm 0.24$ & 0.965 \\
\hline Ischemic heart disease & $39(39)$ & $20(45)$ & $19(34)$ & 0.241 \\
\hline Heart rate, bpm & $71 \pm 15$ & $65 \pm 11$ & $76 \pm 13$ & $<0.001$ \\
\hline Atrial fibrillation & $23(23)$ & $5(11)$ & $18(32)$ & 0.014 \\
\hline NHYA class III or IV & $33(33)$ & $9(20)$ & $23(41)$ & 0.028 \\
\hline Chronic lung disease & $8(8)$ & $2(5)$ & $6(11)$ & 0.259 \\
\hline HFrEF & $54(54)$ & $22(41)$ & $32(59)$ & $<0.001$ \\
\hline Aortic stenosis & $31(31)$ & $11(35)$ & $20(65)$ & $<0.001$ \\
\hline Mitral regurgitation & $15(15)$ & $11(73)$ & $4(27)$ & $<0.001$ \\
\hline \multicolumn{5}{|l|}{ Hemodynamics } \\
\hline Systolic PAP, mmHg & $43 \pm 17$ & $27 \pm 6$ & $55 \pm 13$ & $<0.001$ \\
\hline Diastolic PAP, mmHg & $21 \pm 9$ & $12 \pm 4$ & $27 \pm 7$ & $<0.001$ \\
\hline Mean PAP, mmHg & $29 \pm 12$ & $18 \pm 4$ & $38 \pm 8$ & $<0.001$ \\
\hline PAWP, $\mathrm{mmHg}$ & $19 \pm 8$ & $11 \pm 4$ & $25 \pm 5$ & $<0.001$ \\
\hline Cardiac output, $\mathrm{L} / \mathrm{min}$ & $4.4 \pm 1.3$ & $5.0 \pm 1.4$ & $4.0 \pm 1.1$ & $<0.001$ \\
\hline Cardiac index, $\mathrm{L} / \mathrm{min} / \mathrm{m}^{2}$ & $2.3 \pm 0.7$ & $2.7 \pm 0.7$ & $2.1 \pm 0.7$ & $<0.001$ \\
\hline PVR, Wood Unit & $2.6 \pm 1.8$ & $1.5 \pm 0.7$ & $3.4 \pm 1.9$ & $<0.001$ \\
\hline DPG, mmHg & $1 \pm 0$ & $1 \pm 2$ & $2 \pm 5$ & 0.336 \\
\hline \multicolumn{5}{|l|}{ Echocardiography parameter } \\
\hline TR gradient, mmHg & $32 \pm 17$ & $21 \pm 13$ & $40 \pm 11$ & $<0.001$ \\
\hline \multicolumn{5}{|l|}{ Ungated CT parameters } \\
\hline $\mathrm{dPA}, \mathrm{mm}$ & $29 \pm 5$ & $27 \pm 4$ & $32 \pm 4$ & $<0.001$ \\
\hline $\mathrm{dPA} / \mathrm{AA}$, ratio & $0.89 \pm 0.15$ & $0.80 \pm 0.10$ & $0.96 \pm 0.13$ & $<0.001$ \\
\hline $\mathrm{dPA} / \mathrm{V}$, ratio & $1.07 \pm 0.20$ & $0.98 \pm 0.16$ & $1.14 \pm 0.20$ & $<0.001$ \\
\hline Main PA volume, $\mathrm{ml}$ & $23 \pm 10$ & $19 \pm 8$ & $25 \pm 10$ & $<0.001$ \\
\hline Main PA mean CSA, $\mathrm{mm}^{2}$ & $806 \pm 211$ & $690 \pm 175$ & $898 \pm 193$ & $<0.001$ \\
\hline Main PA length, mm & $27 \pm 6$ & $27 \pm 6$ & $28 \pm 6$ & 0.550 \\
\hline Segmental PA diameter, $\mathrm{mm}$ & $7 \pm 1$ & $7 \pm 1$ & $8 \pm 1$ & 0.061 \\
\hline Segmental ABR, median score* & $1(0-2)$ & $1(0-1)$ & $1(0-2)$ & 0.095 \\
\hline Egg-and-banana sign & $19(19)$ & $5(11)$ & $14(25)$ & 0.084 \\
\hline \multicolumn{5}{|l|}{ ECG-gated CT parameters } \\
\hline $\mathrm{dPA} \max , \mathrm{mm}$ & $31 \pm 5$ & $28 \pm 4$ & $33 \pm 4$ & $<0.001$ \\
\hline dPA/AA max & $0.92 \pm 0.15$ & $0.84 \pm 0.11$ & $0.99 \pm 0.14$ & $<0.001$ \\
\hline $\mathrm{dPA} \min , \mathrm{mm}$ & $28 \pm 5$ & $25 \pm 4$ & $30 \pm 5$ & $<0.001$ \\
\hline $\mathrm{dPA} / \mathrm{AA} \min$ & $0.87 \pm 0.15$ & $0.77 \pm 0.11$ & $0.95 \pm 0.14$ & $<0.001$ \\
\hline Right PA maximal CSA, $\mathrm{mm}^{2}$ & $583 \pm 178$ & $507 \pm 143$ & $643 \pm 180$ & $<0.001$ \\
\hline Right PA minimal CSA, $\mathrm{mm}^{2}$ & $497 \pm 174$ & $401 \pm 125$ & $571 \pm 171$ & $<0.001$ \\
\hline Right PA distensibility, \% & $15.7 \pm 6.9$ & $21.2 \pm 5.4$ & $11.4 \pm 4.4$ & $<0.001$ \\
\hline
\end{tabular}

Values are mean \pm standard deviation, $n(\%)$, or median* $(25$ th -75 th percentile)

$A B R$ pulmonary artery to bronchus ratio; $B M I$ body mass index; $B P M$ beat per minute; $B S A$ body surface area; $C S A$ cross-sectional area; $C T$ computed tomography; $d P A$ diameter of main pulmonary artery; $d P A$ max maximal diameter of main pulmonary artery ; $d P A$ min minimal diameter of main pulmonary artery; $d P A / A A$ ratio between pulmonary artery and aorta diameters; $d P A / A A$ max maximal ratio between pulmonary artery and aorta diameters; $D P A / A A$ min minimal ratio between pulmonary artery and aorta diameters; $d P A / V$ ratio between pulmonary artery and vertebra diameters; $D P G$ diastolic pulmonary gradient; $H F r E F$ heart failure with reduced ejection fraction; NYHA class New York Heart Association functional class; $P A$ pulmonary artery; $P A P$ pulmonary artery pressure; $P A W P$ pulmonary artery wedge pressure; $P H$ pulmonary hypertension; $P V R$ pulmonary vascular resistance; $S D$ standard deviation; $T R$ tricuspid regurgitation

parameters (AUC ranging from 0.54 to 0.83 , DeLong test: $p$ ranging from 0.020 to $<0.001)$. PA distensibility was reproducible between $(r=0.89, p<0.001)$ and within $(r=0.96, p<0.001)$ observers. The correlations between PA distensibility and patient characteristics, PA size, and pulmonary hemodynamics are listed in Table 3 . The correlation between PA distensibility and PAP $(r=-0.72, p<0.001)$ is illustrated in Fig. 3. In multivariable analysis, PA distensibility was independently associated with mean PAP $(\beta=-0.41$, $p<0.001)$, minimal right PA area $(\beta=-0.008, p=0.019)$, and age $(\beta=-0.07, p=0.019)$.

\section{Discussion}

This study shows two main results. First, in patients with HFrEF, aortic stenosis, or primary mitral regurgitation, right PA distensibility was decreased in those with $\mathrm{PH}$ as opposed 
Table 2 Alone and combined echocardiography, ungated CT, and ECG-gated CT parameters: AUC for the diagnosis of $\mathrm{PH}$ and correlation with mean PAP

\begin{tabular}{|c|c|c|c|c|}
\hline & \multicolumn{2}{|l|}{$\mathrm{PH}$ diagnosis } & \multicolumn{2}{|c|}{ Correlation with mean PAP } \\
\hline & $\operatorname{AUC}(95 \% \mathrm{CI})$ & $p$ value & $r$ & $p$ value \\
\hline \multicolumn{5}{|l|}{ Echocardiography } \\
\hline TR gradient, $\mathrm{mmHg}$ & $0.83(0.79-0.90)$ & $<0.001$ & 0.58 & $<0.001$ \\
\hline \multicolumn{5}{|l|}{ Ungated chest $\mathrm{CT}$} \\
\hline $\mathrm{dPA}, \mathrm{mm}$ & $0.81(0.69-0.88)$ & $<0.001$ & 0.55 & $<0.001$ \\
\hline $\mathrm{dPA} / \mathrm{AA}$, ratio & $0.82(0.72-0.89)$ & $<0.001$ & 0.54 & $<0.001$ \\
\hline $\mathrm{dPA} / \mathrm{V}$, ratio & $0.75(0.63-0.83)$ & $<0.001$ & 0.43 & $<0.001$ \\
\hline Main PA volume, $\mathrm{mL}$ & $0.73(0.61-0.82)$ & $<0.001$ & 0.30 & 0.002 \\
\hline Main PA mean CSA, $\mathrm{mm}^{2}$ & $0.80(0.67-0.89)$ & $<0.001$ & 0.48 & $<0.001$ \\
\hline Main PA length, mm & $0.54(0.42-0.65)$ & 0.237 & 0.01 & 0.902 \\
\hline Segmental PA diameter, $\mathrm{mm}$ & $0.59(0.46-0.69)$ & 0.066 & 0.32 & 0.002 \\
\hline Segmental ABR score $(0-4)$ & $0.59(0.48-0.69)$ & 0.044 & 0.22 & 0.028 \\
\hline Egg-and-banana sign & $0.57(0.49-0.63)$ & 0.036 & 0.16 & 0.117 \\
\hline \multicolumn{5}{|l|}{ ECG-gated chest CT } \\
\hline $\mathrm{dPA} \max , \mathrm{mm}$ & $0.77(0.66-0.85)$ & $<0.001$ & 0.45 & $<0.001$ \\
\hline dPA/AA max, ratio & $0.80(0.69-0.87)$ & $<0.001$ & 0.47 & $<0.001$ \\
\hline $\mathrm{dPA} \min , \mathrm{mm}$ & $0.81(0.70-0.88)$ & $<0.001$ & 0.54 & $<0.001$ \\
\hline $\mathrm{dPA} / \mathrm{AA}$ min, ratio & $0.84(0.74-0.90)$ & $<0.001$ & 0.55 & $<0.001$ \\
\hline Right PA max CSA, $\mathrm{mm}^{2}$ & $0.72(0.60-0.80)$ & $<0.001$ & 0.38 & $<0.001$ \\
\hline Right PA min CSA, mm² & $0.80(0.69-0.87)$ & $<0.001$ & 0.49 & $<0.001$ \\
\hline Right PA distensibility, $\%$ & $0.92(0.86-0.96)$ & $<0.001$ & -0.72 & $<0.001$ \\
\hline
\end{tabular}

Abbreviations as in Table 1 to those without $\mathrm{PH}$ and correlated negatively with mean PA pressure. Second, among all tested parameters, PA distensibility performed the best for detecting $\mathrm{PH}$, better than all vascular signs available from ungated CT and TR gradient in echocardiography. These findings deserve further discussion with regard to the diagnostic value of these CT signs and the physiological relationships between PA pressure and PA distensibility.

With regard to the diagnostic value, our study shows that, for detecting PH, PA distensibility performs well with the AUC reaching 0.92 , a value very close to that reported by Revel et al (i.e., 0.95) in mixed types of PH [21]. Most
Fig. 2 ROC curves for PH detection in patients with left heart disease. The AUC of PA distensibility (0.92) at EGC-gated $\mathrm{CT}$ was significantly larger than that of $\mathrm{dPA} / \mathrm{dAA}$ ratio at ungated CT $(0.82, p=0.019)$ and TR gradient at echocardiography $(0.83$, $p=0.020$ )

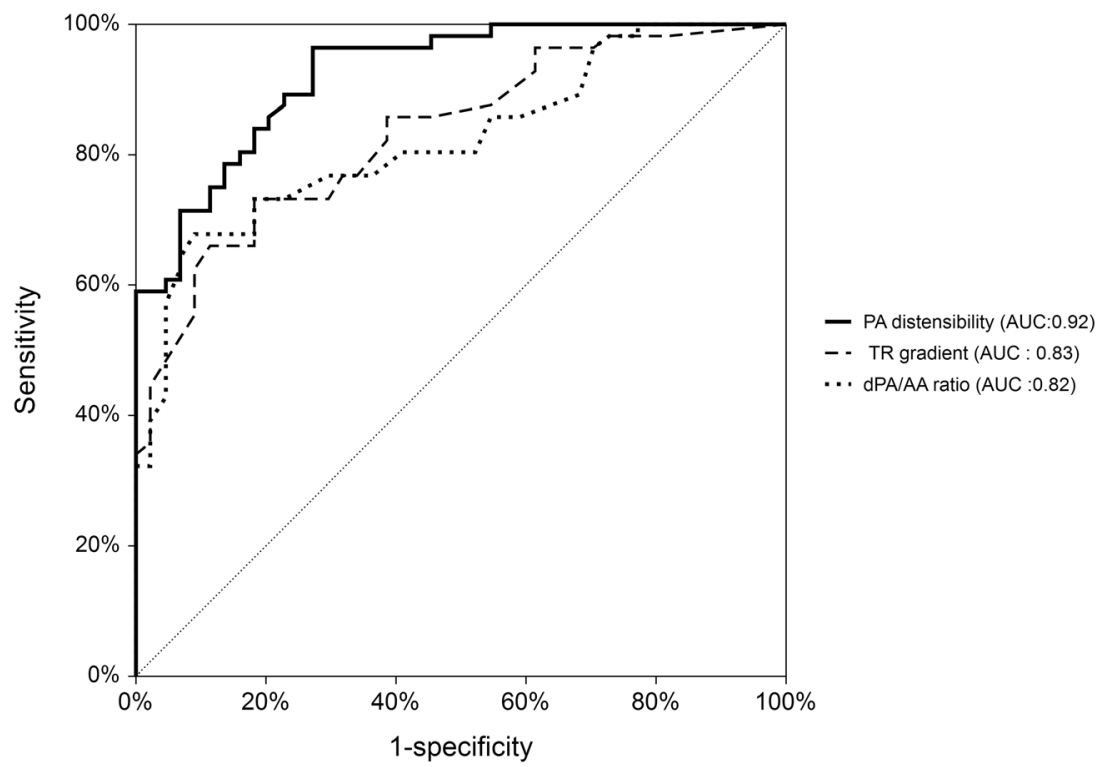


Table 3 Relationship of PA distensibility with patient characteristics, PA size, and hemodynamics

\begin{tabular}{lrr}
\hline & Correlation coefficient $(r)$ & $p$ value \\
\hline Age, years & -0.35 & $<0.001$ \\
BMI, $\mathrm{kg} / \mathrm{m}^{2}$ & 0.16 & 0.111 \\
BSA, ${ }^{2}$ & 0.17 & 0.089 \\
Ischemic heart disease & 0.06 & 0.529 \\
Heart rate, bpm & -0.40 & $<0.001$ \\
Atrial fibrillation & -0.27 & 0.006 \\
Chronic lung disease & -0.11 & 0.289 \\
Mean PAP, mmHg & -0.72 & $<0.001$ \\
PAWP, mmHg & -0.65 & $<0.001$ \\
PVR, WU & -0.59 & $<0.001$ \\
Cardiac index & 0.38 & $<0.001$ \\
Right PA minimal CSA, $\mathrm{mm}^{2}$ & -0.59 & $<0.001$ \\
Right PA maximal CSA, $\mathrm{mm}^{2}$ & -0.42 & $<0.001$ \\
\hline
\end{tabular}

Abbreviations as in Table 1

importantly, PA distensibility performed better than TR gradient in echocardiography and any measurement or sign of proximal and peripheral PA dilatation in ungated CT.

In ungated $\mathrm{CT}, \mathrm{dPA}$ and $\mathrm{APA} / \mathrm{AA}$ had the highest value, but their corresponding AUCs were still lower than that of PA distensibility in ECG-gated CT. Thus, evaluation of PA size changes during cardiac cycle through PA distensibility at gated CT performs better than single PA size measurement at ungated CT.

With regard to physiological relationships, correlations between mean PA pressure and PA distensibility, also called relative area change [24], PA pulsatility [25], or PA strain [26], using either maximal cross-sectional area [21, 27-29]

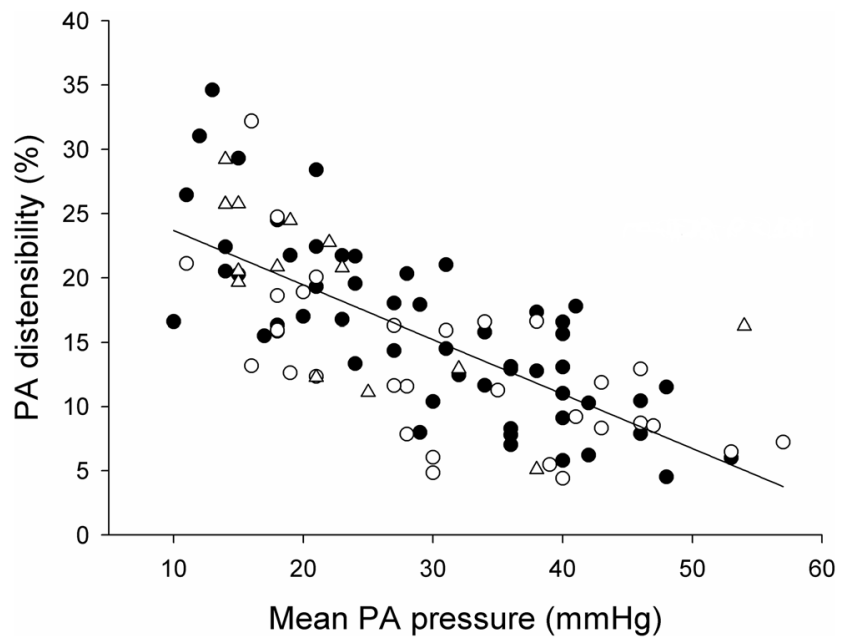

Fig. 3 Correlation between ECG-gated CT-derived right PA distensibility and mean PA pressure $(r=-0.72, p<0.001)$ in patients with HFrEF (black dots), aortic stenosis (white dots), and mitral regurgitation (white triangle) or minimal cross-sectional area [24-26] as denominator, have been recently reported in studies based on ECG-gated CT [21, $27,28]$ and magnetic resonance imaging [24-26, 29] in patients with exclusive [24-27, 29] or almost exclusive [21, 28] precapillary PH. Our study shows that, in patients with left heart disease, PA distensibility is lower in those with PH than in those without PH (11.4\% vs. $21.2 \%)$. These results are within the same order of magnitude as values reported by Revel et al (12.2\% vs. 22.6\%), using ECG-gated CT in 45 patients with mixed types of $\mathrm{PH}$ [21], and by Porter et al ( $10.8 \%$ vs. $17.0 \%)$, using intravascular ultrasound in 30 patients with HFrEF [30]. In fact, CT-derived PA distensibility also correlates with mean PA pressures in PH-LHD. Our study confirms the decrease of PA distensibility in patients with $\mathrm{PH}$ LHD as well, despite the different mechanisms leading to PH.

Although our results show that PA distensibility is a reliable parameter for $\mathrm{PH}$ detection, we acknowledge that PA distensibility computation has several technical issues that might limit its clinical use, including higher radiation exposure associated with retrospective ECG-gated CT, the need for contrast IV injection (although further studies should investigate unenhanced ECG-gated CT in this setting), and the time needed for measurements of cross-sectional area throughout the cardiac cycle. Thus, the added diagnostic value of ECGgated CT should be balanced with these technical issues before considering its potential clinical interest. However, the use of ECG-gated CT before cardiac interventions is growing, and the diagnosis of $\mathrm{PH}$ is very important because of the higher overall mortality rate in patients with $\mathrm{PH}$ than in those without $\mathrm{PH}$ after cardiac procedures, including transcatheter aortic valve implantation [12]. PA distensibility could thus detect PH in patients who have ECG-gated CT as part of their work-up.

Our study has several limitations. First, CT and right heart catheterization were not obtained simultaneously, and we cannot exclude some variations in PA pressure between both procedures. However, patients were stable, and the time interval between $\mathrm{CT}$ and right heart catheterization was quite short. Second, CT acquisition was performed during breath-holding after deep inspiration, while PA pressure was recorded at the end of the expiratory phase during free breathing. Third, the causes of left heart disease were multiple, including HFrEF, mitral regurgitation, and aortic stenosis, each of them carrying different physiological pathways. Our results, therefore, cannot be generalized to any patient with left heart disease. Fourth, we did not evaluate whether the PA distensibility was sensitive to serial changes in mean PA pressure due to ethical reasons regarding repeated $\mathrm{CT}$ and subsequent radiation exposure. Fifth, in accordance with PH guidelines, only the TR gradient was used for $\mathrm{PH}$ detection in echocardiography [1]. However, left ventricular filling pressures may be estimated through other echocardiography parameters that were not evaluated in this study [31]. Sixth, the low temporal 
resolution of CT (140 ms) could have underestimated the actual PA distensibility, especially in patients with high heart rates. Finally, this study was monocentric with a rather small study group.

In conclusion, in patients with severe HFrEF, aortic stenosis, or mitral regurgitation, PA distensibility in ECG-gated CT can detect PH-LHD better than the parameters reflecting PA dilatation in ungated CT or TR gradient in echocardiography.

Funding information The authors state that this work has not received any funding.

\section{Compliance with ethical standards}

Guarantor The scientific guarantor of this publication is Dr. Geoffrey C Colin.

Conflict of interest The authors of this manuscript declare no relationships with any companies, whose products or services may be related to the subject matter of the article.

Statistics and biometry No complex statistical methods were necessary for this paper.

Informed consent Written informed consent was obtained from all subjects (patients) in this study.

Ethical approval Institutional Review Board approval was obtained.

\author{
Methodology \\ - prospective \\ - diagnostic or prognostic study \\ - performed at one institution
}

\section{References}

1. Galie N, Humbert M, Vachiery JL et al (2016) 2015 ESC/ERS guidelines for the diagnosis and treatment of pulmonary hypertension: the Joint Task Force for the Diagnosis and Treatment of Pulmonary Hypertension of the European Society of Cardiology (ESC) and the European Respiratory Society (ERS): endorsed by: Association for European Paediatric and Congenital Cardiology (AEPC), International Society for Heart and Lung Transplantation (ISHLT). Eur Heart J 37(1):67-119

2. Vachiery JL, Adir Y, Barbera JA et al (2013) Pulmonary hypertension due to left heart diseases. J Am Coll Cardiol 62(25 Suppl): D100-D108

3. Ghio S, Gavazzi A, Campana C et al (2001) Independent and additive prognostic value of right ventricular systolic function and pulmonary artery pressure in patients with chronic heart failure. $\mathrm{J}$ Am Coll Cardiol 37(1):183-188

4. Rosenkranz S, Gibbs JS, Wachter R, De Marco T, VonkNoordegraaf A, Vachiery JL (2016) Left ventricular heart failure and pulmonary hypertension. Eur Heart J 37(12):942-954

5. Janda S, Shahidi N, Gin K, Swiston J (2011) Diagnostic accuracy of echocardiography for pulmonary hypertension: a systematic review and meta-analysis. Heart 97(8):612-622
6. Kuriyama K, Gamsu G, Stern RG, Cann CE, Herfkens RJ, Brundage BH (1984) CT-determined pulmonary artery diameters in predicting pulmonary hypertension. Invest Radiol 19(1):16-22

7. Tan RT, Kuzo R, Goodman LR, Siegel R, Haasler GB, Presberg KW (1998) Utility of CT scan evaluation for predicting pulmonary hypertension in patients with parenchymal lung disease. Chest 113(5):1250-1256

8. Ng CS, Wells AU, Padley SP (1999) CT sign of chronic pulmonary arterial hypertension: the ratio of main pulmonary artery to aortic diameter. J Thorac Imaging 14(4):270-278

9. Chan AL, Juarez MM, Shelton DK, MacDonald T, Li CS, Lin TC, Albertson TE (2011) Novel computed tomographic chest metrics to detect pulmonary hypertension. BMC Med Imaging 11:7

10. Davarpanah AH, Hodnett PA, Farrelly CT et al (2011) MDCT bolus tracking data as an adjunct for predicting the diagnosis of pulmonary hypertension and concomitant right-heart failure. AJR Am J Roentgenol 197(5):1064-1072

11. Truong QA, Massaro JM, Rogers IS et al (2012) Reference values for normal pulmonary artery dimensions by noncontrast cardiac computed tomography: the Framingham Heart Study. Circ Cardiovasc Imaging 5(1):147-154

12. Eberhard M, Mastalerz M, Pavicevic J et al (2017) Value of CT signs and measurements as a predictor of pulmonary hypertension and mortality in symptomatic severe aortic valve stenosis. Int $\mathrm{J}$ Cardiovasc Imaging 33(10):1637-1651

13. Colin GC, Gerber BL, de Meester de Ravenstein C et al (2018) Pulmonary hypertension due to left heart disease: diagnostic and prognostic value of CT in chronic systolic heart failure. Eur Radiol 28(11):4643-4653

14. O'Sullivan CJ, Montalbetti M, Zbinden R et al (2018) Screening for pulmonary hypertension with multidetector computed tomography among patients with severe aortic stenosis undergoing transcatheter aortic valve implantation. Front Cardiovase Med 5:63

15. Scelsi CL, Bates WB, Melenevsky YV, Sharma GK, Thomson NB, Keshavamurthy JH (2018) Egg-and-banana sign: a novel diagnostic CT marker for pulmonary hypertension. AJR Am J Roentgenol 210(6):1235-1239

16. Li M, Wang S, Lin W et al (2018) Cardiovascular parameters of chest $\mathrm{CT}$ scan in estimating pulmonary arterial pressure in patients with pulmonary hypertension. Clin Respir J 12(2):572-579

17. Devaraj A, Wells AU, Meister MG, Corte TJ, Wort SJ, Hansell DM (2010) Detection of pulmonary hypertension with multidetector CT and echocardiography alone and in combination. Radiology 254(2): 609-616

18. Spruijt OA, Bogaard HJ, Heijmans MW et al (2015) Predicting pulmonary hypertension with standard computed tomography pulmonary angiography. Int J Cardiovasc Imaging 31(4):871-879

19. Rengier F, Wörz S, Melzig C et al (2016) Automated 3D volumetry of the pulmonary arteries based on magnetic resonance angiography has potential for predicting pulmonary hypertension. PLoS One 11(9):e016251

20. Melzig C, Wörz S, Egenlauf E et al (2019) Combined automated $3 \mathrm{D}$ volumetry by pulmonary CT angiography and echocardiography for detection of pulmonary hypertension. Eur Radiol 29(11): 6059-6068

21. Revel MP, Faivre JB, Remy-Jardin M, Delannoy-Deken V, Duhamel A, Remy J (2009) Pulmonary hypertension: ECG-gated 64-section $\mathrm{CT}$ angiographic evaluation of new functional parameters as diagnostic criteria. Radiology 250(2):558-566

22. Ponikowski P, Voors AA, Anker SD et al (2016) 2016 ESC guidelines for the diagnosis and treatment of acute and chronic heart failure. Eur Heart J 27:2129-2200

23. Stamm G (2012) Collective radiation dose from MDCT: critical review of surveys studies. In: Tack D, Kalra MK, Gevenois PA (eds) Radiation dose from multidetector CT, 2nd edn. SpringerVerlag, Heidelberg, pp 209-229 
24. Tji-Joong Gan C, Lankhaar JW, Westerhof N et al (2007) Noninvasively assessed pulmonary artery stiffness predicts mortality in pulmonary arterial hypertension. Chest 132(6):1906-1912

25. Sanz J, Kariisa M, Dellegrottaglie S et al (2009) Evaluation of pulmonary artery stiffness in pulmonary hypertension with cardiac magnetic resonance. JACC Cardiovasc Imaging 2(3):286-295

26. Sanz J, Kuschnir P, Rius T et al (2007) Pulmonary arterial hypertension: noninvasive detection with phase-contrast MR imaging. Radiology 243(1):70-79

27. Kasai H, Sugiura T, Tanabe N et al (2014) Electrocardiogram-gated 320 -slice multidetector computed tomography for the measurement of pulmonary arterial distensibility in chronic thromboembolic pulmonary hypertension. PLoS One 9(11):e111563

28. Abel E, Jankowski A, Pison C, Luc Bosson J, Bouvaist H, Ferretti GR (2012) Pulmonary artery and right ventricle assessment in pulmonary hypertension: correlation between functional parameters of
ECG-gated CT and right-side heart catheterization. Acta Radiol 53(7):720-727

29. Jardim C, Rochitte CE, Humbert M et al (2007) Pulmonary artery distensibility in pulmonary arterial hypertension: an MRI pilot study. Eur Respir J 29:476-481

30. Porter TR, Taylor DO, Fields J et al (1993) Direct in vivo evaluation of pulmonary arterial pathology in chronic congestive heart failure with catheter-based intravascular ultrasound imaging. Am J Cardiol 71:754-757

31. Andersen OS, Smiseth OA, Dokainish H et al (2017) Estimating left ventricular filling pressure by echocardiography. J Am Coll Cardiol 69(15):1937-1948

Publisher's note Springer Nature remains neutral with regard to jurisdictional claims in published maps and institutional affiliations. 